\title{
1/4 Ke V DIFFUSE BACKGROUND AND THE LOCAL INTERSTELLAR MEDIUM
}

\author{
S.L. Snowden \\ Max-Planck-Institute for Extraterrestrial Physics
}

The $1 / 4 \mathrm{keV}$ diffuse $\mathrm{X}$-ray background (SXRB) is discussed in relation to the local interstellar medium (LISM). The most likely source for these soft $\mathrm{X}$-rays is thermal emission from a hot diffuse plasma. The existence of a non-zero flux from all directions and the short ISM mean free path of these X-rays $\left(10^{20} \mathrm{HI} \mathrm{cm}^{-2}\right)$, coupled with ISM pressure constraints, imply that the plasma has a local component and that it must, at least locally (nearest hundred parsecs), have a large filling factor. Our understanding of the geometry and physical parameters of the LISM is therefore directly tied to our understanding of the SXRB.

Two extreme SXRB source models are: 1) The displacement model; the source plasma is contained within a cavity in the galactic HI surrounding the Sun and the observed negative correlation between soft $\mathrm{X}$-ray flux and $\mathrm{HI}$ is produced entirely by a competition for space. 2) The interspersed model; the emitting plasma and $\mathrm{HI}$ are intermixed with the plasma having no effect on the HI distribution. The observed negative correlation is produced entirely by absorption, or by a combination of absorption and latitude effects due to different scale heights for the hot and cold gas. Observational consequences of these models are discussed, with the results favoring something much closer to 1 ).

With the assumption that the displacement model provides an accurate representation of solar neighborhood, the structure of the LISM is discussed. The local plasma-filled cavity is irregulary shaped and is distended out of the galactic plane with an aspect ratio of $\sim 2.5$ with a mean radius of $\sim 100 \mathrm{pc}$. Some parameters of the plasma are: electron density, $\sim 0.005 \mathrm{~cm}^{-3}$, pressure, $(\mathrm{p} / \mathrm{k}) \sim 9000 \mathrm{~cm}^{-3} \mathrm{~K}$, and temperature, $10^{6.0} \mathrm{~K}$. Finally, the displacement model is shown to be consistent with diverse observations of the LISM. 